九州大学学術情報リポジトリ

Kyushu University Institutional Repository

Introducing Viewpoints of Mechanics into Basic Growth Analysis-(XII) : Applying Sign Reversal of Phase, Space Inversion and Time Reversal to Wave Functions with Negative Amplitude-

Shimojo, Masataka

Laboratory of Animal Feed Science, Division of Animal Science, Department of Animal and Marine Bioresource Sciences, Faculty of Agriculture, Kyushu University

https://doi.org/10.5109/16115

出版情報: 九州大学大学院農学研究院紀要. 54 (2)，pp.357-359，2009-10-29. Faculty of Agriculture, Kyushu University

バージョン：

権利関係 : 


\title{
Introducing Viewpoints of Mechanics into Basic Growth Analysis - (XII) Applying Sign Reversal of Phase, Space Inversion and Time Reversal to Wave Functions with Negative Amplitude -
}

\author{
Masataka SHIMOJO* \\ Laboratory of Animal Feed Science, Division of Animal Science, Department of Animal and Marine \\ Bioresource Sciences, Faculty of Agriculture, Kyushu University, \\ Fukuoka 812-8581, Japan \\ (Received April 30, 2009 and accepted July 13, 2009)
}

\begin{abstract}
This study was conducted to apply sign reversal of phase, space inversion and time reversal to wave functions with negative amplitude. The negative amplitude occurred when \pm signs are given to each of three terms including amplitude when the square root was taken to solve a differential equation. Although a phase shift of $\pi$ changed negative amplitude into positive amplitude, there were some mathematical operations that might be expected to avoid negative amplitude. The results obtained were as follows. Space inversion showed reversing the directions of all three-space coordinates of the wave. Time reversal showed the wave moving backward in time. The product of sign reversal of phase and space inversion or time reversal gave positive amplitude to the wave. Boldly speaking at the risk of making mistake, these mathematical operations suggested that the wave with positive amplitude and sign reversal of phase moved along reversal directions of space and backward in time. They were summarized by the transformation of coordinates from $\left(\boldsymbol{i} r, x, y, z, t,-W_{0}\right)$ to $\left(-\boldsymbol{i} r,-x,-y,-z,-t, W_{0}\right)$. It was suggested from the present study that the negative amplitude in wave functions was hypothetically avoided by applying sign reversal of phase, space inversion and time reversal.
\end{abstract}

\section{INTRODUCTION}

When expanded into the world of complex numbers, wave functions described by complex numbers, as well as basic growth functions described by real numbers, are derived from the same differential equation that is given by introducing growth jerk into basic growth function (Shimojo, 2006, 2007, 2009a). Those wave functions are associated with micro-structures observed in animals and plants (Shimojo et al., 2003c) and vines of plants, and also used for the symbolic representation of field-forage ruminant relationships (Shimojo et al., 2003a, b). It appears, therefore, that wave functions suggest some aspects of animal agriculture. However, similarly to negative weight problem in basic growth functions (Shimojo et al., 2009b), there also occurs a negative amplitude in wave functions. This comes from giving \pm signs to each of three terms including amplitude when the square root is taken to solve the differential equation. The amplitude of a wave normally takes a positive value. The phase shift of $\pi$ changes negative amplitude into positive amplitude. Despite this hard fact, there are mathematical operations of wave functions that might be expected to avoid negative amplitude.

The present study was designed to avoid negative amplitude hypothetically by applying sign reversal of phase, space inversion and time reversal to wave functions with negative amplitude

\footnotetext{
* Corresponding author (E-mail: mshimojo@agr.kyushu-u.ac.jp)
}

APPLYING SIGN REVERSAL OF PHASE, SPACE INVERSION AND TIME REVERSAL TO WAVE FUNCTIONS WITH NEGATIVE AMPLITUDE

(A) Wave functions suggested by growth jerk introduced into basic growth functions

A differential equation for basic growth mechanics including growth jerk is given by a series of the following calculations.

$$
W=W_{0} \cdot \exp \left(r_{W} \cdot t\right)
$$

where $W=$ weight, $t=$ time, $r_{W}=$ relative growth rate of $W, W_{0}=W$ at $t=0$.

Then, absolute growth rate (AGR), growth acceleration (GA) and growth jerk (GJ) are given by

$$
\begin{aligned}
& \mathrm{AGR}=d W / d t=r_{w} \cdot W_{0} \cdot \exp \left(r_{W} \cdot t\right), \\
& \mathrm{GA}=d^{2} W / d t^{2}=r_{W}{ }^{2} \cdot W_{0} \cdot \exp \left(r_{w} \cdot t\right), \\
& \mathrm{GJ}=d^{3} W / d t^{3}={r_{W}}^{3} \cdot W_{0} \cdot \exp \left(r_{W} \cdot t\right),
\end{aligned}
$$

Combining functions (2), (3) and (4) gives

$$
\frac{d^{2} W / d t^{2}}{d W / d t}=\frac{d^{3} W / d t^{3}}{d^{2} W / d t^{2}}=r_{W} .
$$

Therefore, a differential equation for basic growth mechanics is given by

$$
\left(d^{2} W / d t^{2}\right)^{2}=(d W / d t) \cdot\left(d^{3} W / d t^{3}\right)
$$

When expanded into the world of complex numbers, the square root of the right-hand side of differential equa- 
tion (6) is as follows,

$$
\begin{aligned}
& \pm \sqrt{(d W / d t) \cdot\left(d^{3} W / d t^{3}\right)} \\
& = \pm \sqrt{\left(r_{W} \cdot W_{0} \cdot \exp \left(r_{W} \cdot t\right)\right) \cdot\left(r_{W}{ }^{3} \cdot W_{0} \cdot \exp \left(r_{W} \cdot t\right)\right)} \\
& = \pm\left(W_{0}\right) \cdot\left(r_{W}{ }^{2}\right) \cdot\left(\exp \left(r_{W} \cdot t\right)\right) .
\end{aligned}
$$

The negative solution in function (7) occurs only in the world of complex numbers, because in the world of real numbers only positive solution is obtained due to the nature of growth acceleration. Therefore, forcedly giving negative sign at the risk of making mistakes requires the leap into the world of complex numbers (Shimojo, 2006, 2007, 2009a).

As reported by Shimojo (2009a), giving \pm signs to each of three terms in the right-hand side of function (7) requires modifying function (1) as follows in order to conserve differential rules,

$$
\begin{aligned}
& W=\left( \pm W_{0}\right) \cdot\left( \pm \exp \left(\left( \pm r_{W}\right) \cdot t\right)\right), \\
& \Psi=\left( \pm W_{0}\right) \cdot\left( \pm \exp \left(\left( \pm \boldsymbol{i} r_{W}\right) \cdot t\right)\right),
\end{aligned}
$$

where $i$ = imaginary unit, $\Psi=$ wave function.

In the present study, \pm signs are also given to $t$ in functions (8) and (9) as follows,

$$
\begin{aligned}
& W=\left( \pm W_{0}\right) \cdot\left( \pm \exp \left(\left( \pm r_{W}\right) \cdot( \pm t)\right)\right), \\
& \Psi=\left( \pm W_{0}\right) \cdot\left( \pm \exp \left(\left( \pm \boldsymbol{i} r_{W}\right) \cdot( \pm t)\right)\right) .
\end{aligned}
$$

Function (10), which is basic growth function, was investigated for the hypothetic avoidance of negative weight in the preceding paper (Shimojo et al., 2009b) in the same issue. Therefore, wave function (11) is taken up in the present study. Function (11) includes 16 wave functions. Each wave function, which is a solution to differential equation (6), has its own meaning (Shimojo, 2009a). In wave function (11), $W_{0}$ is amplitude and $r_{W}$ is phase component. Theses are absolutely different from those of growth function (10), where $W_{0}$ is weight and $r_{W}$ is relative growth rate. This is a kind of qualitative leap from growth functions to wave functions.

In the present study, the following wave function (12) is taken out of function (11) for the issue of negative amplitude,

$$
\Psi=\left( \pm W_{0}\right) \cdot \exp \left(\boldsymbol{i} r_{w} \cdot t\right) .
$$

Wave function (12) includes negative amplitude as well as positive amplitude and there is interference between them, resulting in a disappearance of wave phenomenon. However, amplitude normally takes a positive value. If there occurs a phase shift of $\pi$, then this changes negative amplitude into positive amplitude as follows,

$$
\begin{aligned}
\left(-W_{0}\right) \cdot \exp \left(\boldsymbol{i} r_{W} \cdot t\right) & \rightarrow\left(-W_{0}\right) \cdot \exp \left(\boldsymbol{i}\left(r_{W} \cdot t+\pi\right)\right) \\
& =\left(-W_{0}\right) \cdot \exp \left(\boldsymbol{i} r_{W} \cdot t\right) \cdot \exp (\boldsymbol{i} \pi)
\end{aligned}
$$

$$
\begin{aligned}
& =\left(-W_{\mathbf{0}}\right) \cdot \exp \left(\boldsymbol{i} r_{W} \cdot t\right) \cdot(-1) \\
& =W_{\mathbf{0}} \cdot \exp \left(\boldsymbol{i} r_{W} \cdot t\right) .
\end{aligned}
$$

Despite this hard fact, there are some mathematical operations that might be expected to avoid negative amplitude. This will be tried in the following section.

\section{(B) Applying sign reversal of phase, space inver- sion and time reversal to wave function with nega- tive amplitude}

In this section, the negative amplitude will be hypothetically avoided by applying sign reversal of phase, space inversion and time reversal to wave function with negative amplitude.

Wave function with negative amplitude is given by

$$
\Psi=\left(-W_{0}\right) \cdot \exp \left(\boldsymbol{i} r_{W} \cdot t\right)
$$

There is a correspondence between wave function (11) and basic growth function (10). Therefore, according to the following twelve basic growth functions based on space and time (Shimojo et al., 2009b),

$$
\begin{aligned}
& W=W_{\mathbf{0}} \cdot \exp \left(r_{W} \cdot t\right), W=W_{\mathbf{0}} \cdot \exp \left(r_{\boldsymbol{a}} \cdot x\right) \\
& W=W_{\mathbf{0}} \cdot \exp \left(r_{\boldsymbol{b}} \cdot y\right), W=W_{\mathbf{0}} \cdot \exp \left(r_{\boldsymbol{c}} \cdot z\right) \\
& W=\left(-W_{\mathbf{0}}\right) \cdot \exp \left(r_{\boldsymbol{W}} \cdot t\right), W=\left(-W_{\mathbf{0}}\right) \cdot \exp \left(r_{\boldsymbol{a}} \cdot x\right), \\
& W=\left(-W_{\mathbf{0}}\right) \cdot \exp \left(r_{\boldsymbol{b}} \cdot y\right), W=\left(-W_{\mathbf{0}}\right) \cdot \exp \left(r_{\boldsymbol{c}} \cdot z\right), \\
& W=W_{\mathbf{0}} \cdot \exp \left(\left(-r_{W}\right) \cdot(-t)\right), W=W_{\mathbf{0}} \cdot \exp \left(\left(-r_{\boldsymbol{a}}\right) \cdot(-x)\right), \\
& W=W_{\mathbf{0}} \cdot \exp \left(\left(-r_{\boldsymbol{b}}\right) \cdot(-y)\right), W=W_{\mathbf{0}} \cdot \exp \left(\left(-r_{\boldsymbol{c}}\right) \cdot(-z)\right)
\end{aligned}
$$

the corresponding twelve wave functions based on space and time are given as follows,

$$
\begin{aligned}
& \Psi=W_{0} \cdot \exp \left(\boldsymbol{i} r_{W} \cdot t\right), \Psi=W_{0} \cdot \exp \left(\boldsymbol{i} r_{\boldsymbol{a}} \cdot x\right), \\
& \Psi=W_{0} \cdot \exp \left(\boldsymbol{i} r_{\boldsymbol{b}} \cdot y\right), \Psi=W_{0} \cdot \exp \left(\boldsymbol{i} r_{\boldsymbol{c}} \cdot z\right), \\
& \Psi=\left(-W_{0}\right) \cdot \exp \left(\boldsymbol{i} r_{W} \cdot t\right), \Psi=\left(-W_{\mathbf{0}}\right) \cdot \exp \left(\boldsymbol{i} r_{\boldsymbol{a}} \cdot x\right), \\
& \Psi=\left(-W_{0}\right) \cdot \exp \left(\boldsymbol{i} r_{\boldsymbol{b}} \cdot y\right), \Psi=\left(-W_{0}\right) \cdot \exp \left(\boldsymbol{i} r_{\boldsymbol{c}} \cdot z\right),(19) \\
& \Psi=W_{0} \cdot \exp \left(\left(-\boldsymbol{i} r_{W}\right) \cdot(-t)\right), \Psi=W_{0} \cdot \exp \left(\left(-\boldsymbol{i} r_{\boldsymbol{a}}\right) \cdot(-x)\right), \\
& \Psi=W_{0} \cdot \exp \left(\left(-\boldsymbol{i} r_{\boldsymbol{b}}\right) \cdot(-y)\right), \\
& \Psi=W_{\mathbf{0}} \cdot \exp \left(\left(-\boldsymbol{i} r_{\boldsymbol{c}}\right) \cdot(-z)\right) .
\end{aligned}
$$

It is shown that negative amplitude in function (19) is changed into positive amplitude in function (20) by sign reversal of phase, space inversion and time reversal. Function (20) expresses the wave whose phase, space and time are absolutely opposite to those of the wave in function (18). Space inversion shows reversing the 
directions of all three space coordinates of the wave. Time reversal shows the wave moving backward in time. The product of sign reversal of phase and space inversion or time reversal gives positive amplitude to the wave as shown in function (20). Boldly speaking at the risk of making mistake, wave function (20) suggests that the wave with positive amplitude and sign reversal of phase moves along reversal directions of space and backward in time. These mathematical operations are summarized by the following transformation of coordinates in wave functions,

$$
\left(\boldsymbol{i} r, x, y, z, t,-W_{0}\right) \rightarrow\left(-\boldsymbol{i} r,-x,-y,-z,-t, W_{0}\right) .
$$

However, animal agricultural meaning that may be derived from these mathematical operations remains to be investigated.

\section{(C) Space-time relationships suggested through wave in the world of complex numbers}

The following space-time relationship is derived from wave function (18)

$$
\boldsymbol{i} r_{a} \cdot x=\boldsymbol{i} r_{b} \cdot y=\boldsymbol{i} r_{c} \cdot z=\boldsymbol{i} r_{W} \cdot t .
$$

Relation (22) suggests at the risk of making mistakes that three-dimensional space and time are treated equally and $r$ shows a relative resistance of wave to move toward each of four dimensions in the world of complex numbers.

\section{(D) Conclusions}

It is suggested from the present study that the negative amplitude in wave functions is hypothetically avoided by applying sign reversal of phase, space inversion and time reversal.

\section{REFERENCES}

Shimojo, M., K. Ikeda, Y. Asano, R. Ishiwaka, T. Shao, H. Sato, M. Tobisa, Y. Nakano, N. Ohba, Y. Yano and Y. Masuda 2003a A symbolic representation of field-forage-ruminant relationships using polar form on the complex plane. J. Fac. Agr., Kyushu Univ., 47: 359-366

Shimojo, M., Y. Asano, K. Ikeda, R. Ishiwaka, T. Shao, H. Sato, M. Tobisa, Y. Nakano, N. Ohba, Y. Yano and Y. Masuda 2003b Complex representation of field-forage-ruminant relationships using symmetric properties of Euler's formula. J. Fac. Agr., Kyushu Univ., 47: 367-372

Shimojo, M., Y. Asano, K. Ikeda, R. Ishiwaka, T. Shao, H. Sato, M. Tobisa, Y. Nakano, N. Ohba, Y. Yano and Y. Masuda 2003c A stereographic representation of Euler's formula to show spirals and topological similarities to micro-structures in ruminants and forages. J. Fac. Agr., Kyushu Univ., 48 $71-76$

Shimojo, M. 2006 Introducing viewpoints of mechanics into basic growth analysis - (II) Relative growth rate compared with energy in wave function -. J. Fac. Agr., Kyushu Univ., 51: $289-291$

Shimojo, M. 2007 Introducing viewpoints of mechanics into basic growth analysis - (V) Problems derived from introducing growth jerk -. J. Fac. Agr., Kyushu Univ., 52: 77-79

Shimojo, M. 2009a Introducing viewpoints of mechanics into basic growth analysis - (X) Wave functions in comparison and relationship with growth functions -. J. Fac. Agr., Kyushu Univ., 54: 141-145

Shimojo, M., T. Shao, J. Tanoue, H. Kakihara, C. Sata, H. Fukudome, R. Ishiwaka, Y. Asano, Y. Nakano, M. Tobisa and Y. Masuda 2009b Introducing viewpoints of mechanics into basic growth analysis - (XI) Negative weight problem in basic growth functions and its hypothetic avoidance by sign reversal of relative growth rate, space inversion and time reversal -. J. Fac. Agr., Kyushu Univ., 54: 353-355 